\title{
RATIONALE OF ANTIBIOTIC PRESCRIPTION IN PATIENTS VISITING A TERTIARY CARE SOUTH INDIAN HOSPITAL
}

\author{
MOHAMED AHMED ${ }^{1 *}$, PAWAN KUMAR ${ }^{2}$, D.V. KISHORE ${ }^{3}$, PRABHAT KUMAR ${ }^{4}$, RUBEENA KAUSER ${ }^{5}$, MOAZAM ALI $^{5}$ \\ ${ }^{1}$ Research Scholar, Singhania University, Pacheri Bari, Jhunjhunu, Rajasthan. ${ }^{2}$ School of Pharmacy and Medical Sciences, Singhania \\ University, Pacheri Bari, Jhunjhunu, Rajasthan. ${ }^{3}$ Department of Pharmacology, Shadan college of Pharmacy, Hyderabad, Telangana. \\ ${ }^{4}$ Department of Microbiology, Singhania University, Pacheri Bari, Rajasthan. ${ }^{5}$ Department of Pharmacy Practice, Shadan College of \\ Pharmacy, Hyderabad, Telangana. Email: moin086@gmail.com
}

Received: 15 October 2021, Revised and Accepted: 27 October 2021

\section{ABSTRACT}

Objective: Antimicrobials are the class of drugs that are used irrationally in most cases leading to rise in instances of antimicrobial resistance altering the effect of such agents. Antimicrobial resistance has become a critical issue universally nerving the need to monitor the utilization pattern and rationality in prescribing of antibiotics. This helps in selection of most appropriate antibiotic for the specific patient and achieving the goals of the therapy.

Methods: A prospective cross-sectional study with a sample size of 600 participants was conducted in department of general medicine of a tertiary care hospital. Inpatients prescribed with antibiotics were included in the study and their case sheets were reviewed to analyzing the prescribing pattern. The medication usage pattern was then assessed for rationality in prescribing was evaluated. The results obtained were statistically analyzed using SPSS Software.

Results: It is noted that a more number of males participated and the greatest number of the patients were from the age group of 31-45. The diagnosis found in the majority of the patients was respiratory tract infection followed by others. On scrutinizing the prescriptions, it was noted that cephalosporins and penicillins were most often prescribed, and on an average single, antibiotic was frequently used with the preferred route of administration in most of the prescriptions being injection route. Though many of the antibiotics were prescribed empirically, it was observed that $59 \%$ of them were most appropriately dosed and maximum patients that are around 57\% were cured from their illnesses.

Conclusion: This study provided important baseline information on antimicrobial use within a large tertiary care teaching hospital and identified potential targets for future antimicrobial stewardship programs. The culture and sensitivity testing suggested that the drug resistance was more for most commonly prescribed antibiotics in the hospital. Increased targeted prescribing based on sensitivity tests will bring down the high use of empiric broad-spectrum antibiotic use.

Keywords: Antibiotics, Rationality, Microbial Resistance, Stewardship, utilization.

(c) 2021 The Authors. Published by Innovare Academic Sciences Pvt Ltd. This is an open access article under the CC BY license (http://creativecommons.org/ licenses/by/4.0/) DOI: http://dx.doi.org/10.22159/ajpcr.2021v14i1.36082. Journal homepage: https://innovareacademics.in/journals/index.php/ajpcr

\section{INTRODUCTION}

Antimicrobials are the significant class of drugs that are prescribed by the physicians against various bacterial infections. They are produced by microorganisms naturally or may be produced synthetically in laboratories. Antibiotics act by either killing the microorganisms that is bactericidal agents or suppress their growth that is bacteriostatic agents [1]

Infection was a major cause of morbidity and mortality, before the development of antibiotics. The treatment of infections faced a great challenge during those periods. Later in 1928, the discovery of Penicillin, a beta lactam antibiotic, by Alexander Fleming opened up the golden era of antibiotics. It marked a revolution in the treatment of infectious diseases and stimulated new efforts to synthesize newer antibiotics. The period between 1950s and 1970s is considered the golden era of discovery of novel antibiotic classes, with very few classes discovered since then [2].

This class of drugs has evolved the course of modern medicine and is widely used in the patients admitted to the intensive care department; however, there has also been increase in the usage of antibiotics irrationally [2].

Inadequate medical training, lack of diagnostic facilities, widespread availability and usage of irrational fixed dose antimicrobial medication combinations, and neglecting the basic principles of antimicrobial selection and administration are few common factors that contribute to irrational antimicrobial use [3].

Undiscriminating and overuse of antibiotics is a global issue particularly in developing countries with predominance of infectious diseases as a result of which there is an increased incidence of resistance to most common bacteria thereby subsiding the effect of antibiotic, increased treatment cost, longer hospital stay, and adverse drug reactions [4].

Physicians as well as self -medication by patients, liberally using these drugs has led to increasing concern over the risk of development of resistance to antibiotics, known as Antimicrobial Resistance [5]. AMR has now become a serious public health problem limiting the prescriber's choice of specific antibiotics and forcing them to choose a drug which may be expensive, moderately effective against the infection or may even is toxic therefore increasing the morbidity and mortality [6] Such increasing resistance to antibiotics around the world alarms the need to improve the surveillance of usage of antibiotics by assessing their prescribing patterns and to strictly adhere to rationale prescribing of antibiotics and its use [7].

Rational use of drugs means that the patient receives medication appropriate to their clinical needs, in doses that meet the individual requirement for an adequate period of time, and at an economical 
price to the individual and overall to the community [8]. The "Rule of Right" - right medicine in the right manner (dose, route, frequency, and duration of administration) in the right patient at right cost - must be followed while using antibiotics [3]. Therefore, appropriate and safe use of antibiotics in healthcare system is more important not only for economic reasons, but also it plays a key role in achieving the quality care for patients [9].

Quantifying the extent to which irrational use of drugs take place is the first step toward decreasing it. The World Health Organization (WHO) in collaboration with the International Network of Rational Use of Drugs (INRUD) in the 1990s had laid down a set of indicators to estimate the utilization of drugs by healthcare facilities. They are particularly used in three general areas of primary care to measure the performance of rational drug use and are known as core drug indicators. They are prescribing indicators, Patient care indicators and facility indicators. These indicators have been successfully implemented in many developing countries because of its proven

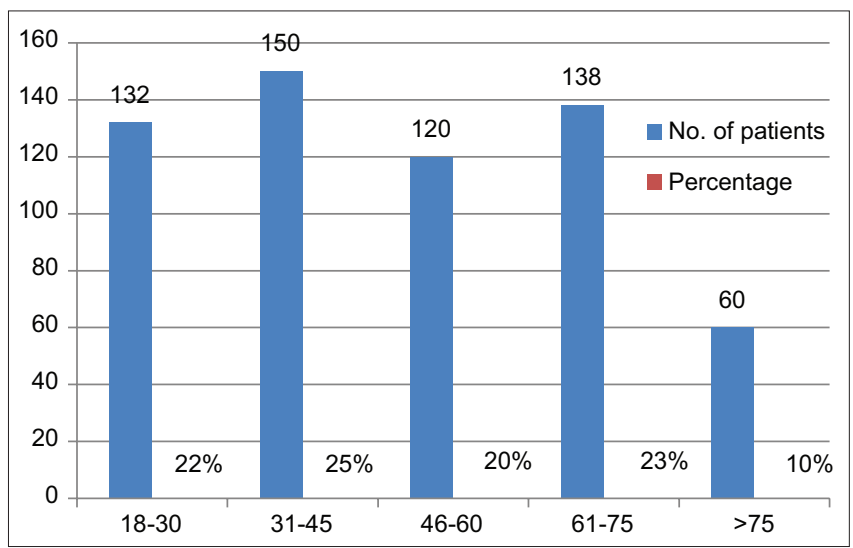

Fig. 1: Age wise distribution among the patients in the study

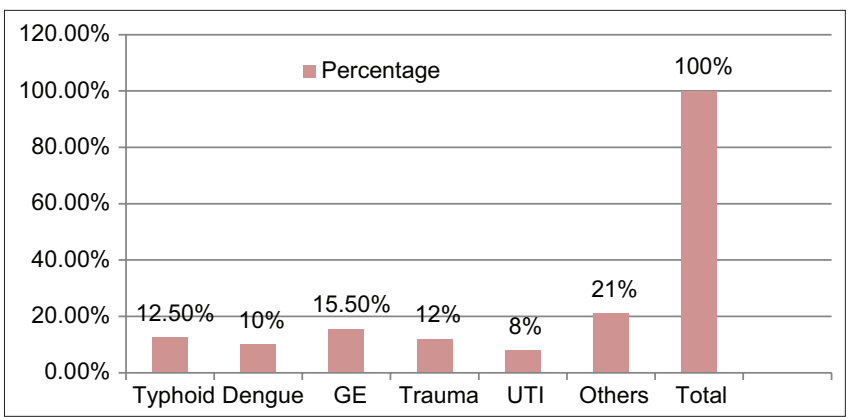

Fig. 2: Prescription differentiation based on diagnosis

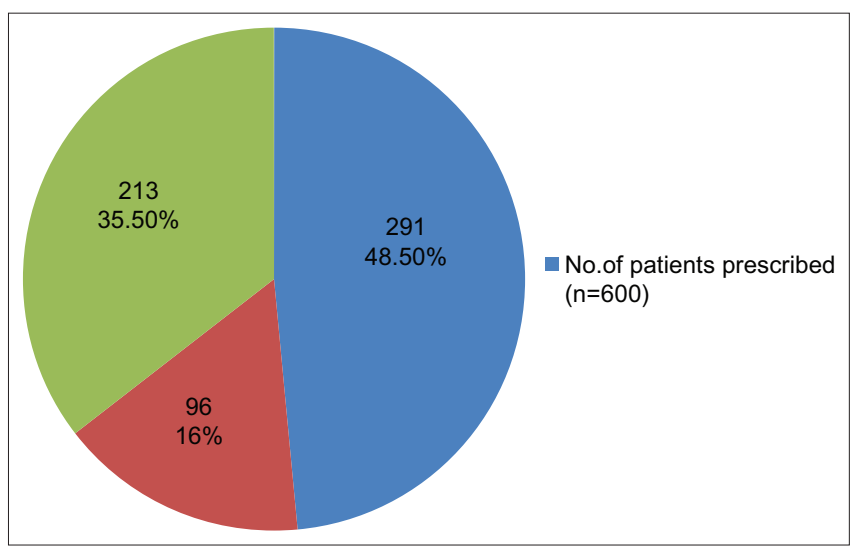

Fig. 3: Reasons for antibiotic prescription feasibility and applicability and hence effectively increase the evaluation of drug usage patterns [4].

NICE Guidelines are the recommendations that focus on educating individuals about how to properly use antimicrobial drugs (including antibiotics) and the risks of overuse and misuse. It also covers infection prevention and control techniques that can reduce the need

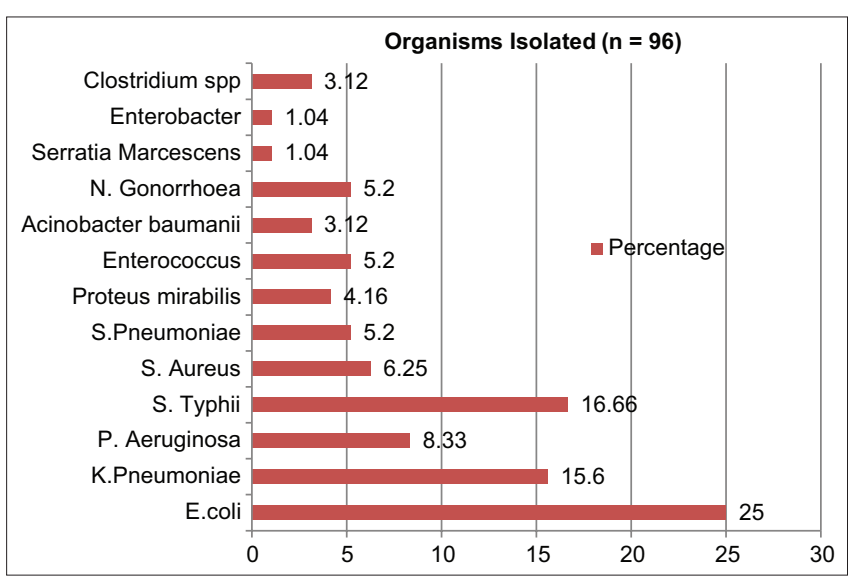

Fig. 4: Organisms isolated

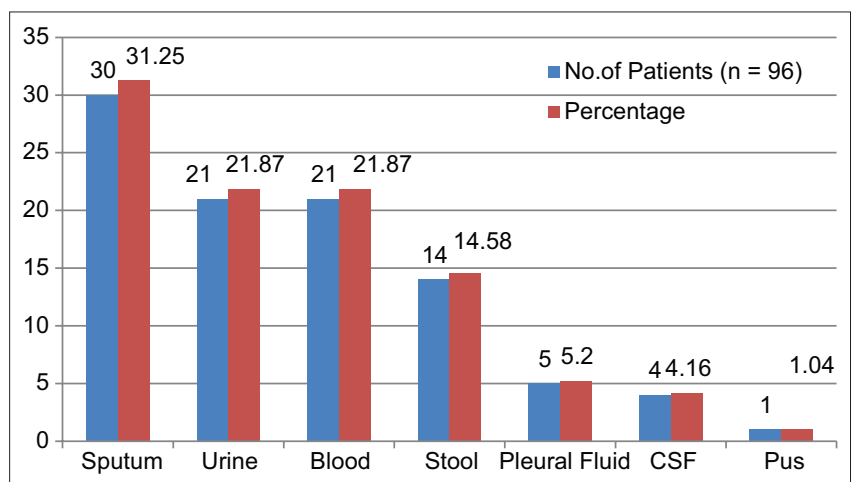

Fig. 5: Specimen sample used for culture sensitivity

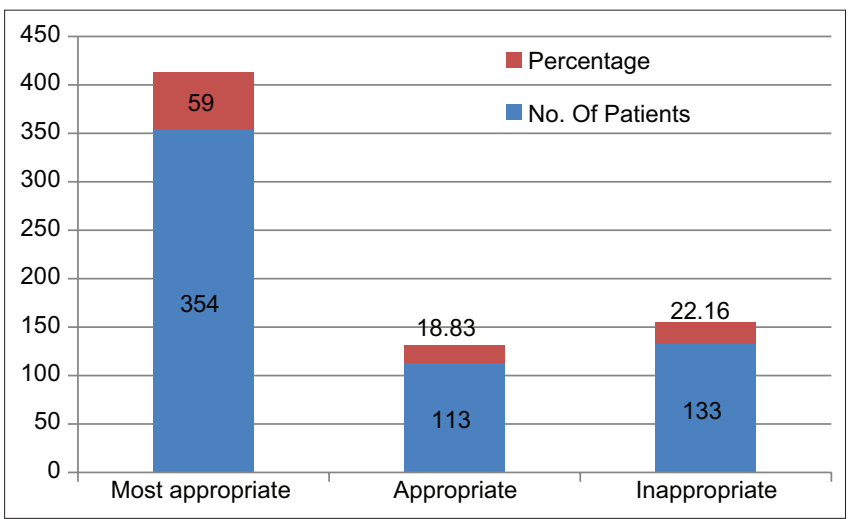

Fig. 6: Appropriateness of antibiotic therapy

Table 1: Gender wise distribution among the patients in the study

\begin{tabular}{lll}
\hline Gender & No of patients & Percentage \\
\hline Male & 348 & $58 \%$ \\
Female & 252 & $42 \%$ \\
Total & 600 & $100 \%$ \\
\hline
\end{tabular}


Table 2: Class and type of antibiotics prescribed

\begin{tabular}{llll}
\hline Class of antibiotics prescribed & Type of the drug & Number of prescriptions & Prescription percentage \\
\hline Cephalosporins & Ceftriaxone & $36.64 \%$ \\
& $\begin{array}{l}\text { Cefoperazone } \\
\text { Cefixime }\end{array}$ & 306 & \\
Cefuroxime & & \\
& Cefpodoxime & & $11.49 \%$ \\
Lluoroquinolones & $\begin{array}{l}\text { Levofloxacin } \\
\text { Ciprofloxacin }\end{array}$ & 96 & $7.42 \%$ \\
Ofloxacin & & $4.91 \%$ \\
Antiprotozoals & Metronidazole & 62 & $35.08 \%$ \\
Macrolides & Clarythromycin & 41 & $3.23 \%$ \\
Nenicillins & Azithromycin & & $0.83 \%$ \\
Aminoglycosides & Piperacillin & 293 & $0.35 \%$ \\
Oxazolidinones & Ampicillin & & $100 \%$ \\
Lincosamide & Amoxicillin & 27 & \\
Total & Amikacin & 7 & \\
\hline
\end{tabular}

Table 3: Therapeutic outcomes of antibiotics

\begin{tabular}{llll}
\hline S. No. & $\begin{array}{l}\text { Therapeutic } \\
\text { outcomes }\end{array}$ & $\begin{array}{l}\text { No. of } \\
\text { patients }(\mathbf{n}=\mathbf{6 0 0})\end{array}$ & Percentage \\
\hline 1 & Cured & 342 & 57 \\
2 & Controlled & 192 & 32 \\
3 & No improvement & 66 & 11 \\
\hline
\end{tabular}

for antimicrobials and the spread of infection to others. Its goal is to alter people's behavior to minimize antimicrobial resistance and the propagation of resistant bacteria [10].

The goal of prescribing pattern research is to track, assess, and advise changes to practitioners' prescribing behaviors to make medical care more reasonable and cost-effective [11] Antibiotic consumption patterns must be understood in order to handle difficulties that occur from various antibiotic usages constructively. It is critical that institutions and hospitals have an antibiotic policy in place and that individual prescribers make the best decisions possible. Prescribers can benefit from highly representative data, which can help them utilize antibiotics more wisely and improve the quality of their patients' treatment [9]

\section{METHODS}

A prospective, cross-sectional study was conducted for 6 months in Department of General Medicine of a 720 bedded tertiary care hospital from January 2019 to December 2019. Both inpatients and outpatients of General Medicine who were prescribed with at least one antimicrobial agent were included in the study as per the inclusion criteria. A total of 600 patients who met the inclusion criteria were selected for the study. The patients were well informed about the study, and written informed consent was obtained before including them in the study. Data were collected and recorded in a specially designed case collection form. A guideline regarding rational use of antibiotics was developed after reviewing relevant literatures, electronic database and using NICE guidelines with respect to dose, indication, frequency and duration of antibiotics. Results were statistically analyzed descriptively using SPSS software.

\section{Inclusion criteria}

- Patients above 18 years of age.

- Prescriptions with Antibiotic usage were included.

\section{Exclusion criteria}

- Pediatrics below 12 years was excluded.

- Pregnant, lactating women were excluded.

\section{RESULTS}

Out of 600 patients included in the study majority of them were males that are around $58 \%$ of the participants whereas females accounted for $42 \%$ of the participants.

Among the 600 patients who participated in the study, 132 of them were under the age group of 13-30, 150 of them were under 31-45, 120 of them were under 46-60, 138 were under the group $61-75$ and only 60 of them were above the age of 75 years, maximum number of patients were in the age group of $31-45$ years that is $25 \%$ of the population.

On evaluating the usage pattern of antibiotics, it is observed that cephalosporins is the class of drugs that was mostly prescribed with a prescription percentage of $36.64 \%$ followed by penicillins accounting for $35.08 \%$ of prescriptions and fluoroquinolones accounting for $11.49 \%$ of prescriptions. Other classes of antibiotics such as antiprotozoals (7.42\%), macrolides (4.91\%), aminoglycosides (3.23\%), oxazolidinones $(0.83 \%)$, and lincosamide $(0.35 \%)$ were less frequently prescribed.

Among the 600 prescriptions, the most common diagnosis was found to be respiratory tract infection in $21 \%$ of prescriptions followed by gastroenteritis in $15.50 \%$ of prescriptions, typhoid in $12.50 \%$ of prescriptions, and trauma in $12 \%$ of prescriptions. Diseases such as dengue and urinary tract infections were comparatively less commonly encountered in only $10 \%$ and $8 \%$ of prescriptions, respectively. Other diagnosis accounted for around $21 \%$.

On evaluation of reasons for prescribing antibiotics, it is noted that majority of them were prescribed empirically that is $48.5 \%$, and $16 \%$ of them were prescribed based on bacteriologically proven infections while $35.5 \%$ were prescribed for non-bacteriologically proven infections.

Out of 600 patients, 342 (57\%) of them had their aliments cured and around 192 (32\%) showed a control over their infections with the use of antibiotics, however $66(11 \%)$ of them had no improvement at all.

On conducting culture sensitivity test E. coli was mostly isolated organism in around 24 patients followed by $S$. Typhi in 16 patients, K. pnuemoniae in 15 patients, $P$. aeruginosa in 8 patients and S. aureus in six patients.

S. pneumoniae, N. gonorrhoea and Enterococcus were isolated in five patients. Proteus mirabilis was found in four patients. Three patients 
each were infected with Acinobacter baumanii and Clostridium spp. Serratia marcescens and Enterobacter were isolated from only one patient each.

Sputum sample was widely used with $31.25 \%$ followed by urine and blood sample at $21.87 \%$, stool (14.58\%), pleural fluid $(5.2 \%)$, CSF (4.16\%), and pus (1.04\%).

When the appropriateness of dosing of antibiotics was evaluated maximum prescriptions that is 354 (59\%) were most appropriate whereas 113 (18.83\%) prescriptions had appropriate dosing, whereas $22.16 \%$ were found to be inappropriate, $(22.16 \%)$ were found to be inappropriate.

\section{DISCUSSION}

In our study, male patients were predominant and the age of the patients ranged between 31 and 45 years were found to be high. The results revealed that out of 600 prescriptions, 835 antibiotics were prescribed to the study population. The most commonly prescribed antibiotics were cephalosporins (36.64\%), followed by Penicillins (35.08\%). These results were similar to a study done by Raphael [6]. 78\% of prescriptions had 1 antibiotic followed by $20 \%$ of prescriptions having 2 antibiotics. A similar study was done by Abdel Salam Mohamed Elfaki and reported that cephalosporins to be used in $1 / 3^{\text {rd }}$ of the prescriptions especially ceftriaxone [12,13]. 59\% (354) of prescriptions were most appropriate whereas $22.16 \%$ (133) were found to be inappropriate. Culture sensitivity test found that E.coli $(\mathrm{n}=96)$ was most isolated organism in $25 \%$ patients followed by $S$. Typhi in $16.66 \%$ patients. A study by Ambili ramesh found that E.coli was most identified organism [9].

\section{CONCLUSION}

This study analyzed 600 prescriptions and found that physicians used the drugs from the WHO EML. About 54\% prescriptions were appropriate whereas other remaining prescriptions were found to be inappropriate which shows that there is a need to develop empiric antibiotic guidelines as to guide clinicians in appropriate antibiotic prescribing. This study provided important baseline information on antimicrobial use within a large tertiary care teaching hospital and identified potential targets for future antimicrobial stewardship programs. The culture and sensitivity testing suggested that the drug resistance was more for most commonly prescribed antibiotics in the hospital. Increased targeted prescribing based on sensitivity tests will bring down the high use of empiric broadspectrum antibiotic use. Irrational use of drugs can be detected by frequent prescription auditing.

\section{REFERENCES}

1. Hashemi S, Nasrollah A, Rajabi M. Irrational antibiotic prescribing: A local issue or global concern? EXCLI J 2013;12:384-95.

2. Silva CD, Silva M Jr. Strategies for appropriate antibiotic use in intensive care unit. Einstein (Sao Paulo) 2015;13:448-53

3. DesaiSV. Rational use of antibiotics in clinical practice: Pharmacological consideration. J Integr Health Sci 2019;7:1-2.

4. Rajalingam B, Alex AS, Godwin A, Cherian C, Cyriac C. Assessment of rational use of antibiotics in a private tertiary care teaching hospital. Indian J Pharm Pract 2016;9:14-8.

5. Kourkouta L, Kotsiftopoulos $\mathrm{CH}$, Papageorgiou M, Iliadis $\mathrm{CH}$, Monios A. The rational use of antibiotics medicine. J Healthc Commun 2017;2:3.

6. Kayambankadzanja RK, Lihaka M, Barratt-Due A, Kachingwe M, Kumwenda W, Lester R, et al. The use of antibiotics in the intensive care unit of a tertiary hospital in Malawi. BMC Infect Dis 2020;20:776.

7. National Treatment Guidelines for Antimicrobial Use in Infectious Diseases. Version 1.0 National Centre for Disease Control, Directorate General of Health Services Ministry of Health and Family Welfare Government of India; 2016. Available from: https://ncdc.gov.in/ WriteReadData/1892s/File622.pdf

8. Sengupta S, Pal B, Bala T. Rational use of antibiotics and its guidelines in surgical patients-a retropspective study. Surg Int J Surg Trauma Orthop 2017;3:33-7.

9. Remesh A, Salim AM, Gayathri UN, Retnavally KG. Antibiotics prescribing pattern in the in-patient departments of a tertiary care hospital. Pharm Pract 2013;4:71-6.

10. Antimicrobial Stewardship: Changing Risk-related Behaviours in the General Population. Available from: https://www.nice.org.uk/guidance/ng63

11. Shankar RP, Partha P, Shenoy NK, Easow JM, Brahmadathan KN. Prescribing patterns of antibiotics and sensitivity patterns of common microorganisms in the internal medicine ward of a teaching hospital in Western Nepal: A prospective study. Ann Clin Microbiol Antimicrob 2003;2:7.

12. Rational for Antibiotics Guidelines, Mangesh Tiwaskar, Tanuja Manohar, Chapter2. Available from: http://apiindia.org/wp-content/ uploads/pdf/medicine update_2017/mu_002.pdf

13. Elfaki AM. Assessment of antibiotics prescription in hospitalized patients at Elobeid hospital. Sudan J Med Sci 2010;4:1-7. 